OPEN ACCESS

Edited by:

Liana Fattore,

Consiglio Nazionale delle Ricerche

(CNR), Italy

Reviewed by:

Cristiano Bertolucci,

University of Ferrara, Italy

Diego A. Golombek,

Universidad Nacional de Quilmes (UNQ), Argentina

*Correspondence: Da-long Ren rend/@ustc.edu.cn Bing $\mathrm{Hu}$

bhu@ustc.edu.cn

Received: 08 December 2017 Accepted: 01 March 2018 Published: 13 March 2018

Citation: Huang $D$, Wang $M$, Yin W, Ma Y, Wang $H$, Xue $T$, Ren D and Hu B (2018) Zebrafish Lacking Circadian Gene per2 Exhibit Visual Function Deficiency.

Front. Behav. Neurosci. 12:53. doi: 10.3389/fnbeh.2018.00053

\section{Zebrafish Lacking Circadian Gene per2 Exhibit Visual Function Deficiency}

\author{
Deng-feng Huang ${ }^{1}$, Ming-yong Wang ${ }^{2}$, Wu Yin ${ }^{1}$, Yu-qian $\mathrm{Ma}^{3}$, Han Wang ${ }^{2}$, Tian Xue ${ }^{3}$, \\ Da-long Ren ${ }^{1 *}$ and Bing $\mathrm{Hu}^{1 *}$
}

${ }^{1}$ Hefei National Laboratory for Physical Sciences at the Microscale and CAS Key Laboratory of Brain Function \& Disease, School of Life Sciences, University of Science and Technology of China, Hefei, China, ${ }^{2}$ School of Biology \& Basic Medical Sciences, Medical College, Soochow University, Suzhou, China, ${ }^{3}$ Neurodegenerative Disorder Research Center, University of Science and Technology of China, Hefei, China

The retina has an intrinsic circadian clock, but the importance of this clock for vision is unknown. Zebrafish offer many advantages for studying vertebrate vision and circadian rhythm. Here, we explored the role of zebrafish per2, a light-regulated gene, in visual behavior and the underlying mechanisms. We observed that per2 mutant zebrafish larvae showed decreased contrast sensitivity and visual acuity using optokinetic response (OKR) assays. Using a visual motor response (VMR) assay, we observed normal OFF responses but abnormal ON responses in mutant zebrafish larvae. Immunofluorescence showed that mutants had a normal morphology of cone photoreceptor cells and retinal organization. However, electron microscopy showed that per2 mutants displayed abnormal and decreased photoreceptor ribbon synapses with arciform density, which resulted in retinal ON pathway defect. We also examined the expression of three cone opsins by quantitative real-time PCR (qRT-PCR), and the expression of long-wave-sensitive opsin (opn1/W) and short-wave-sensitive opsin (opn1sw) was reduced in mutant zebrafish larvae. qRT-PCR analyses also showed a down-regulation of the clock genes cry $1 \mathrm{ba}$ and bmal1b in the adult eye of per2 mutant zebrafish. This study identified a mechanism by which a clock gene affects visual function and defined important roles of per2 in retinal information processing.

Keywords: per2, optokinetic response, contrast sensitivity, ribbon synapses, visual motor response, opsin

\section{INTRODUCTION}

The circadian clock is an endogenous oscillator that drives daily rhythms in many living systems. In mammals, the master pacemaker is located in the suprachiasmatic nucleus, which generates a regular rhythm with an approximate 24-h period (Reppert and Weaver, 2002; Ko and Takahashi, 2006). The retinal clock was the first extra-SCN circadian oscillator discovered in mammals and is an endogenous circadian clock that regulates many physiological processes within the neural retina (Tosini et al., 2008). A variety of visual behaviors, including photoreceptor cell disk shedding (Besharse, 1982), retinomotor movements (Besharse, 1982; Douglas et al., 1992), and photoreceptor synaptic ribbons (Wagner, 1973), display robust day-night rhythms. The circadian clock is thought to adjust the visual sensitivity to ambient light levels, although the involvement of specific clock genes is poorly understood, particularly in zebrafish. 
Based on extensive research, this clock consists of interlocking feedback loops in which the CLOCK-BMAL1 transcription factor drives the expression of Per and Cry genes (Ko and Takahashi, 2006). According to a previous study, deletion of the core clock component bmall leads to defects in the physiological processing of vision in mice (Storch et al., 2007). Mice lacking per 1 and per 2 show significant alterations in the distribution of cone photoreceptors (Ait-Hmyed et al., 2013). Moreover, in the absence of Rev-erb $\alpha$, mouse retinas modify the scotopic threshold responses and increase pupillary constriction, thereby increasing the sensitivity to light (Hakkari et al., 2016). These studies defined the important role of the clock component in eye vision research.

Zebrafish offer many advantages for studying vertebrate vision and circadian rhythmicity, and the zebrafish retina is similar in structure and function to those of other vertebrates, including mammals (Easter and Nicola, 1996). The molecular mechanisms of circadian rhythm generation in zebrafish appear to have much in common with the more extensively studied mammalian system, although the details are different (Cahill, 2002). Zebrafish homologs of three per genes have also been identified: two per 1 homologs (per $1 a$ and per $1 b$ ) and a single copy of the per 2 and per 3 genes. Zebrafish per 2 is a light-regulated gene, and its expression is significantly damped under constant darkness (Vatine et al., 2009). Similar to cryla, per2 has also been suggested to act as a photoreceptor and is crucial for the aanat 2 rhythms established in the zebrafish pineal gland (Ziv et al., 2005; Tamai et al., 2007). Furthermore, a more recent study of PER2 loss-of-function fish generated by the TALEN method revealed PER2 may serve both as a transcriptional coactivator and a corepressor for Ror/Reverb response element enhancers and E-box enhancers, respectively (Wang et al., 2015).

Currently, little is known about mechanisms underlying how clock gene modulates retinal physiological responses to light. In this study, we used zebrafish as a model to investigate the function of per2. We used a variety of anatomical, molecular biological and behavioral approaches to explore the retinal phenotype of zebrafish larvae that lacked the per2 gene. Data showed that per $2^{-/-}$zebrafish larvae displayed abnormal photoreceptor ribbon synapses and decreased ribbon synapses with arciform density, which led to visual behavior deficiency compared with the wild-type (WT) fish.

\section{MATERIALS AND METHODS}

\section{Animals and Maintenance}

Per2 mutant zebrafish were obtained using the TALEN genomeediting tool (Wang et al., 2015). WT AB strain and per2 mutant zebrafish were maintained and raised on a 14-h/10-h light/dark (LD) cycle at $28^{\circ} \mathrm{C}$ as previously described (Ren et al., 2015). For dark/dark (DD) experiments, the embryos were removed from the LD cycle at 20:00 $\mathrm{h}$ on the day before the experiment and were then kept under DD conditions. All animal manipulations were conducted in strict accordance with the guidelines and regulations set forth by the University of Science and Technology of China (USTC) Animal Resources Center and University Animal Care and Use Committee. The protocol was approved by the Committee on the Ethics of Animal Experiments of the USTC (Permit Number: USTCACUC1103013).

\section{Optokinetic Response Assays}

The optokinetic response (OKR) test was performed as previously described (Mueller and Neuhauss, 2010). Briefly, we used the computer software LabVIEW to generate a sine-wave grating that was projected by an LCD projector (NEC 280+; NEC Corporation, Japan). Zebrafish larvae were immobilized in $6 \%$ methylcellulose in a $35-\mathrm{mm}$ petri dish and placed dorsal side up above a small infrared light. With the rotating grating patterns presented around the larva, the elicited eye movement was recorded in real time by an infrared-sensitive CCD camera (TCA-1.3BW; Nanjing, China). WT and per 2 mutant larvae were stimulated with a constant angular velocity of 7.5 degree/s and a fixed spatial frequency (SF) of 0.04 cycle/degree. To measure the visual acuity, the SF was presented with $0.02,0.04,0.06$ and 0.08 cycle/degree. We used the gain (ratio of eye velocity and stimulus velocity) of the OKR to measure contrast sensitivity (Rinner et al., 2005).

\section{Visual Motor Response Assay}

The visual motor response (VMR) assay was based on a published design (Emran et al., 2007, 2008). The assay was conducted inside a ZebraBox system (ViewPoint Life Sciences, Lyon, France). Mutants and AB-WT were each placed in one of 48 wells of a 96-well plate. Before the actual experiment, the 96-well plate with the larvae was dark-adapted in the ZebraBox system for $3.5 \mathrm{~h}$ to acclimatize the animals. The light change (on or off) was abrupt and instantaneous. Larval movement was summarized as the fraction of frames in which a larva displayed movement in each second. The data were processed and analyzed using custom PERL software and Visual Basic Macros for Microsoft Excel.

\section{RNA Extraction and Quantitative Real-Time PCR (qRT-PCR)}

Total RNAs were extracted from approximately 30 larvae of the homozygous per 2 or WT fish at different times (ZT $0=$ lights on) of the day and night under LD or DD conditions, and from 3-month adult retinas using RNAiso Plus (Takara) reagent as the manufacturer's protocol, respectively. Specifically, adult zebrafish retinas were rapidly peeled from the eye tissues with forceps after deeply anesthesia with MS222, and the tissue was frozen at $-80^{\circ} \mathrm{C}$ until further assays. Then, RNAs were reverse transcribed into cDNA using a HiScript ${ }^{\circledR}$ II 1st Strand cDNA Synthesis Kit (Vazyme, Jiangsu, Nanjing, China) according to the manufacturer's protocol. Gene expression was analyzed by qPCR using SYBR Green Master Mix (Vazyme, Jiangsu, NanJing, China) on a LightCycler ${ }^{\circledR} 96$ System (Roche Life Science). The PCR thermal profiles were 40 cycles of $10 \mathrm{~s}$ at $95^{\circ} \mathrm{C}$ and $30 \mathrm{~s}$ at $60^{\circ} \mathrm{C}$. Two housekeeping genes ( $\beta$-actin, $\left.r p l 13 a\right)$ were assessed for stability in adult retinas; $\beta$-actin was more stable and was used for all subsequent analyses. All results were normalized to the expression level of the housekeeping gene $\beta$-actin. The gene-specific primers for per $1 b$, per 2 , per 3, bmal $1 b$, and $c r y 1 b a$ have been described previously (Wang et al., 2015). Primers for clock1a, cacan 1fa, synj1, opn1lw, opn1mw, opn1sw were designed 
TABLE 1 | Primer used in the study.

\begin{tabular}{|c|c|c|}
\hline Gene & Primer sequence & Accession no \\
\hline clock1a & TITGTTGCCACATGCTCCG & NM_130957 \\
\hline cacna1fa & $\begin{array}{l}\text { CCCCTAGAAGCACGCCTATG } \\
\text { CCACTTGCTGGGTAAGGGAG }\end{array}$ & XM_021478483.1 \\
\hline synj1 & $\begin{array}{l}\text { TACCTGCTCCTCTGATGCCT } \\
\text { AACCTGAGGATTGCTCCTGC }\end{array}$ & NM_001007030.2 \\
\hline opn1/w & $\begin{array}{l}\text { TGGAGCAGATACTGGCCTCAT } \\
\text { GGGTCCTCGCTTCCACTGA }\end{array}$ & NM_001313715.1 \\
\hline opn1mw & $\begin{array}{l}\text { GCTGCCACTITGCATACCC } \\
\text { TGTCACTTCCCTCTCAGCCT }\end{array}$ & NM_131253.2 \\
\hline opn1sw & $\begin{array}{l}\text { GTTCGATGGAAGCGGCAATG } \\
\text { ACAGGCGGTACCAATGATCC }\end{array}$ & NM_131319.1 \\
\hline
\end{tabular}

using Primer3 (Rozen and Skaletsky, 2000) and are listed in Table 1. The relative levels of each sample were calculated by the $2^{-\Delta \Delta C T}$ method (Livak and Schmittgen, 2001).

\section{Immunohistochemistry}

Immunohistochemistry was performed as described previously (Jia et al., 2014). For zpr1 immunofluorescence staining, the fish were fixed at $4^{\circ} \mathrm{C}$ for $3 \mathrm{~h}$ with $4 \%$ PFA in PBS ( $\mathrm{pH} 7.4$ ), rinsed with PBS (three times, $5 \mathrm{~min}$ each), and equilibrated in $30 \%$ sucrose in PBS overnight. Then, the samples were transferred into O.C.T. (Tissue-Tek) and sectioned at $10 \mu \mathrm{m}$ on a cryostat (Leica CM1950). The sections were incubated overnight at $4{ }^{\circ} \mathrm{C}$, followed by blocking solution ( $1 \%$ bovine serum albumin, $0.4 \%$ Triton X-100 and 8\% goat serotonin in PBS) for $40 \mathrm{~min}$ and then incubation with zpr-1 antibody (mouse, 1:500; Abcam, Cambridge, MA, USA) for $2 \mathrm{~h}$ at room temperature. After washing with PBS (three times, $5 \mathrm{~min}$ each), the samples were incubated for $2 \mathrm{~h}$ at $4^{\circ} \mathrm{C}$ with Alexa488-conjugated goat anti-mouse (1:1000 dilution, Invitrogen) in blocking solution, counterstained with propidium iodide (PI), and then mounted with $80 \%$ glycerol in PBS for fluorescence microscopy.

\section{Electron Microscopy}

Tissues were processed as described previously (Allwardt et al., 2001). Briefly, zebrafish were fixed in the morning. Whole embryos and larvae were anesthetized and placed into primary fixative for $15 \mathrm{~min}$ at $4^{\circ} \mathrm{C}$. The primary fixative was prepared fresh daily and consisted of $1 \%$ paraformaldehyde, $1.6 \%$ glutaraldehyde, and 3\% sucrose in $0.06 \mathrm{M}$ phosphate buffer, $\mathrm{pH}$ 7.4. The tissue was rinsed and post-fixed in $2 \%$ osmium tetroxide in phosphate buffer for $2 \mathrm{~h}$ at $4^{\circ} \mathrm{C}$. After rinsing, samples were dehydrated in a graded series of ethanol-water mixture and infiltrated with epon/araldite resin. The tissue was cured for $72 \mathrm{~h}$ at $60^{\circ} \mathrm{C}$. Ultrathin $(50 \mathrm{~nm})$ transverse sections of the retina from larvae of both zebrafish genotypes were stained with uranyl acetate and lead citrate. Sections were viewed and photographed with an FEI Tecnai Spirit (120 kV TEM) transmission electron microscope.

\section{Statistical Analyses}

Data are shown as the mean \pm SEM. Statistical significance was determined using a two-tailed unpaired Student's $t$ test or one-way ANOVA or two-way ANOVA with a significance level of $P<0.05$. A repeated-measure adjustment was employed when appropriate (e.g., daily gene expression profiles). Two-way ANOVA followed by post hoc Bonferroni test was applied when needed (e.g., OKR). In particular, we used three independent samples for analysis and three repeated independent measurements for the gene expression experiment, with similar results. For the JTK-CYCLE analysis, each parameter was recorded and analyzed as previously described by R (Hughes et al., 2010). Statistical analysis was performed with GraphPad Prism5 software (San Diego, CA, USA).

\section{RESULTS}

\section{per2 Deficiency Affects Zebrafish Larvae Visual Behavior}

To determine whether visual responsiveness was altered in the per 2 mutant zebrafish, we recorded the OKR of 5 days post-fertilization (dpf) larvae of the two genotypes at different times of day and night. Compared with the WT, per $2^{-/-}$
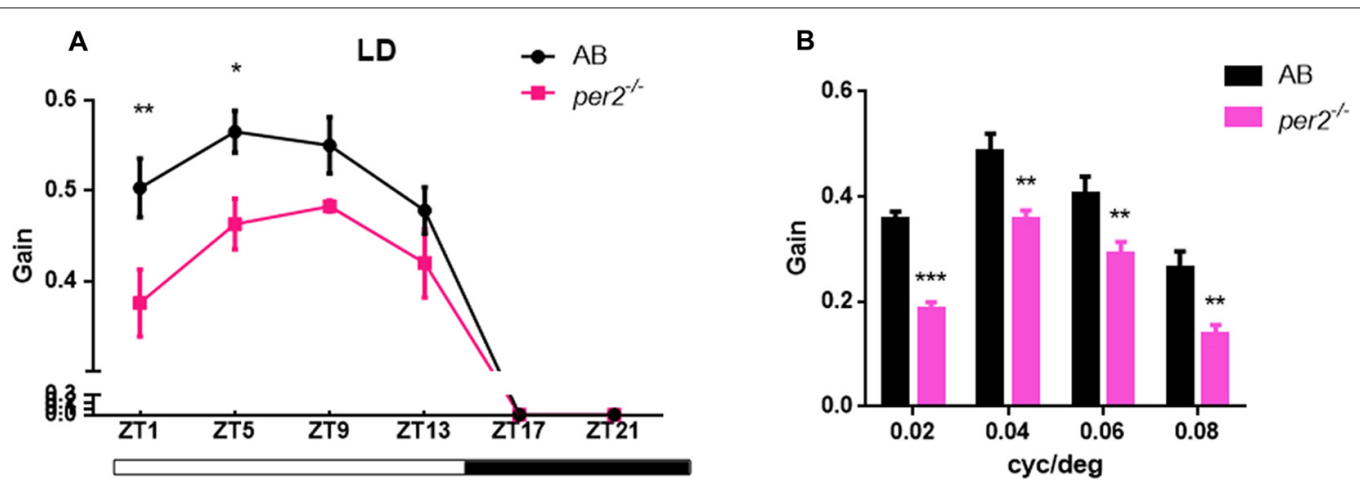

FIGURE 1 | per2 mutant zebrafish larvae showed optokinetic response (OKR) deficiency. (A) OKRs of AB and per2 mutant during the course of a day under the light/dark (LD) condition (ZTO light on, ZT14 light off; Contrast $=0.6, n=10 ; Z \mathrm{~T}: F_{(5,85)}=124.8, P<0.0001 ;$ Genotype: $\left.F_{(1,85)}=12.01, P=0.0008\right)$. (B) OKRs of AB and per2 mutant under different spatial frequency (SF; contrast $=0.6, n=10$; cycle/degree: $F_{(3,40)}=30.21, P<0.0001 ;$ Genotype: $\left.F_{(1,40)}=61.65, P<0.0001\right)$. Data from wild type (WT) and per2 mutant were compared using two-way ANOVA followed by a post hoc Bonferroni test. ${ }^{*} P<0.05$, ${ }^{* *} P<0.01$ and ${ }^{* * *} P<0.001$ compared with the WT. Data represent the mean \pm SEM. 
zebrafish larvae displayed attenuated contrast sensitivity during the day (Figure 1A). Two-way ANOVA showed significant effects of genotype $\left(F_{(1,85)}=12.01, P=0.0008\right)$ and $\mathrm{ZT}$ $\left(F_{(5,85)}=124.8, P<0.0001\right)$. The data also indicated that per $2^{-/-}$ zebrafish maintained an OKR circadian rhythm. Another crucial function of the visual system is its ability to distinguish two separate objects at a given angular distance, referred to as visual acuity, and it is influenced by numerous optical and neuronal features of the visual system (Haug et al., 2010). By altering the SF, we found that per $2^{-/-}$mutant larvae showed a significant reduction in gain either at high or low SF (Figure 1B). Two-way ANOVA showed significant effects of genotype $\left(F_{(1,40)}=61.65, P<0.0001\right)$. These results suggested that the mutation of clock gene per 2 caused contrast sensitivity and visual acuity deficiency. We conducted another experiment for visual detection using the VMR, which is a primitive startle response displayed by larvae within seconds after the onset and offset of light (Emran et al., 2007; Zhang et al., 2016). For the light-on VMR, the per 2 mutant fish responded weakly at light onset at both $5 \mathrm{dpf}$ (Figure 2A) and $6 \mathrm{dpf}$ (Figure 2C). At $5 \mathrm{dpf}$, the distance moved variability in $1 \mathrm{~s}$ at light-on was $2.41 \pm 1.94 \mathrm{~mm}$ in WT vs. $0.50 \pm 0.27 \mathrm{~mm}$ in per 2 mutants $(N=48, P<0.01$, two-tailed unpaired $t$-test; Figure 2E). At $6 \mathrm{dpf}$, the distance moved variability in $1 \mathrm{~s}$ at light-on was $2.44 \pm 1.90 \mathrm{~mm}$ in WT vs. $0.84 \pm 0.28 \mathrm{~mm}$ in per2 mutants $(N=48, P<0.01$, two-tailed unpaired $t$-test; Figure 2E). For the light-off VMR, both $\mathrm{WT}$ and per2 mutant fish responded similarly at $5 \mathrm{dpf}$ (Figure 2B) and $6 \mathrm{dpf}$ (Figure 2D). WT and mutant genotypes sharply increased their activity immediately after the cessation of light.

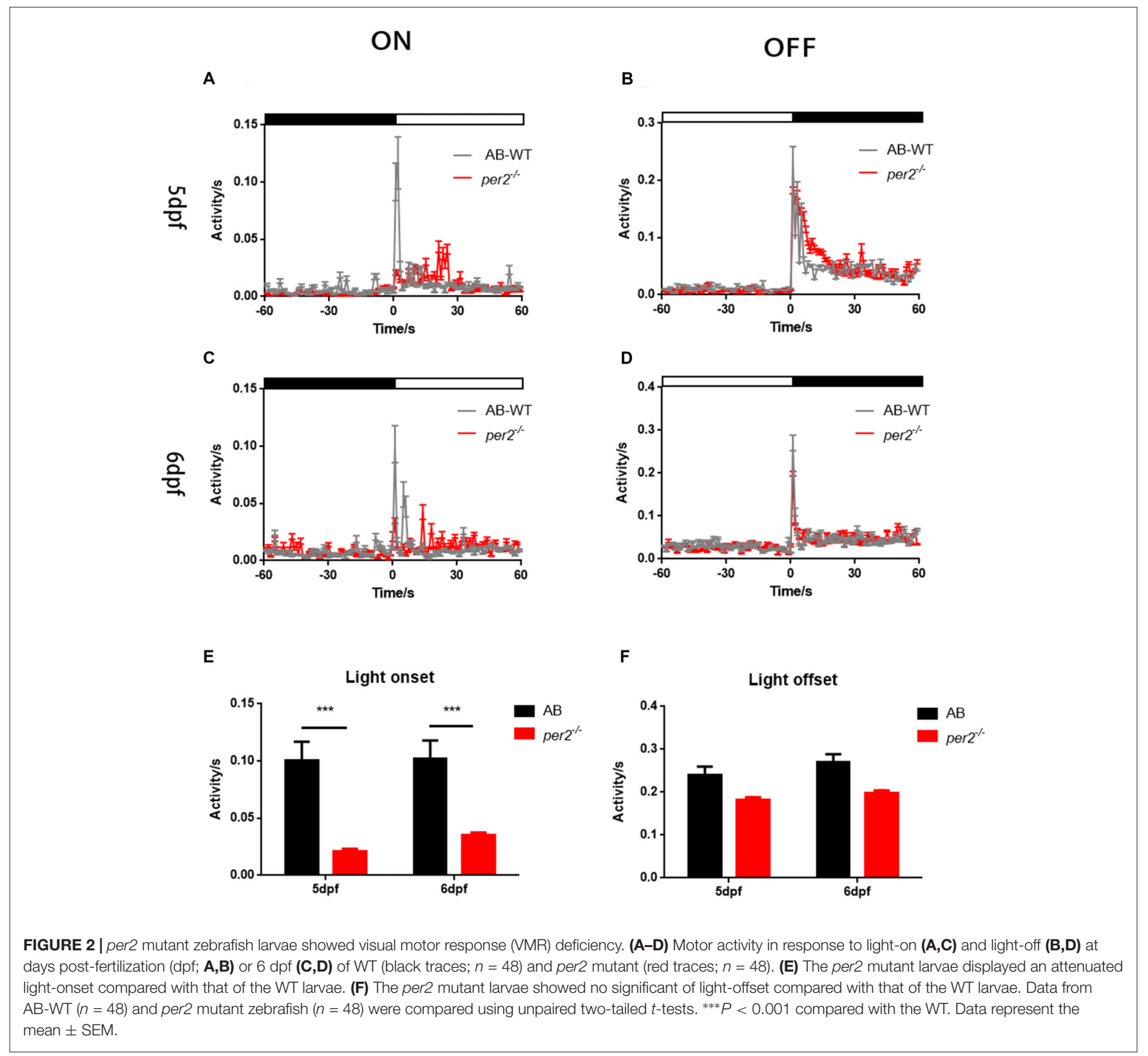


At $5 \mathrm{dpf}$, the distance moved variability in $1 \mathrm{~s}$ at light-off was $5.74 \pm 2.38 \mathrm{~mm}$ in WT vs. $4.38 \pm 0.68 \mathrm{~mm}$ in per2 mutants $(N=48$; Figure $2 \mathrm{~F})$. At $6 \mathrm{dpf}$, the distance moved variability in $1 \mathrm{~s}$ at light-off was $6.49 \pm 2.13 \mathrm{~mm}$ in WT vs. $4.77 \pm 0.62 \mathrm{~mm}$ in per 2 mutants $(N=48$; Figure $2 \mathrm{~F})$. These results indicated that the per 2 mutant fish readily detected the light-off but responded weakly to the light-on; thus, per2 is important for the visual behavior response of zebrafish larvae.

\section{per2-/- Zebrafish Larvae Exhibit Abnormal Photoreceptor Ribbon Synapses Compared With Wild-Type Larvae}

Retinal structural changes might be relevant to the effect of attenuated visual functions. To determine whether retinal development was defective in per $2^{-/-}$mutant larvae, we studied retinal morphology in per $2^{-/}$and WT larvae by histologic staining and electron microscopy. The data showed that per $2^{-/-}$mutant embryos had relatively normal numbers of photoreceptors at $5 \mathrm{dpf}$ based on the immunostaining of cryosections with zpr1 (Figures 3A,B), which marks red-green cones (Larison and Bremiller, 1990).

Because per 2 mutant larvae displayed a light-on VMR defect, we investigated whether this visual defect in mutants was the result of abnormal synaptic ribbons (Allwardt et al., 2001). Retinas were studied by electron microscopy at ZT5, which was the time at which the visual function was obviously defected (Figure 1A). Compared with the WT retina, pedicles in the per 2 mutant had the same basic, normal morphology. However, in mutants, the synaptic ribbons were not close to postsynaptic processes and were rarely associated with an arciform density and a presynaptic membrane (Figure 3C); the synaptic ribbons usually appeared to be "floating" in the cytoplasm (Figure 3D). The data also showed the WT larvae (Figure 3E) had more arciform synaptic ribbons than the mutants (Figure 3F). To quantify the pedicle abnormalities of the per $2^{-/-}$mutants, we studied 40 transversely sectioned pedicles from WT and mutant retinas, as previously reported (Allwardt et al., 2001). The results are shown in Table 2. The number of ribbons per pedicle (row 3) was not significantly different between the WT and mutant populations $(1.40 \pm 0.55$ in the WT vs. $1.55 \pm 0.7$ in mutants, $P=0.345$; two-tailed unpaired $t$-test). We also conducted an ultrastructural analysis to assess how many ribbons were associated with an arciform density. Of the WT ribbons, $73.2 \%$ had an associated arciform density in the plane of section, whereas $38.7 \%$ of the mutant ribbons were associated (row 4). These data confirmed that the retinal synapses were affected by the per 2 mutation.

A previous study showed that wud (cacnalfa) mutants lack synaptic ribbons and that wud is essential for the development of synapses. Synaptojanin 1 (synj1) mutants have floating synaptic ribbons (Jia et al., 2014). Thus, we checked the mRNA expression of two genes in the larval retina. We found that the expression of both cacnalfa $(P=0.007$ compared with the WT; Figure 3G) and synj1 ( $P=0.022$ compared with the WT; Figure $3 \mathbf{H}$ ) was significantly reduced in
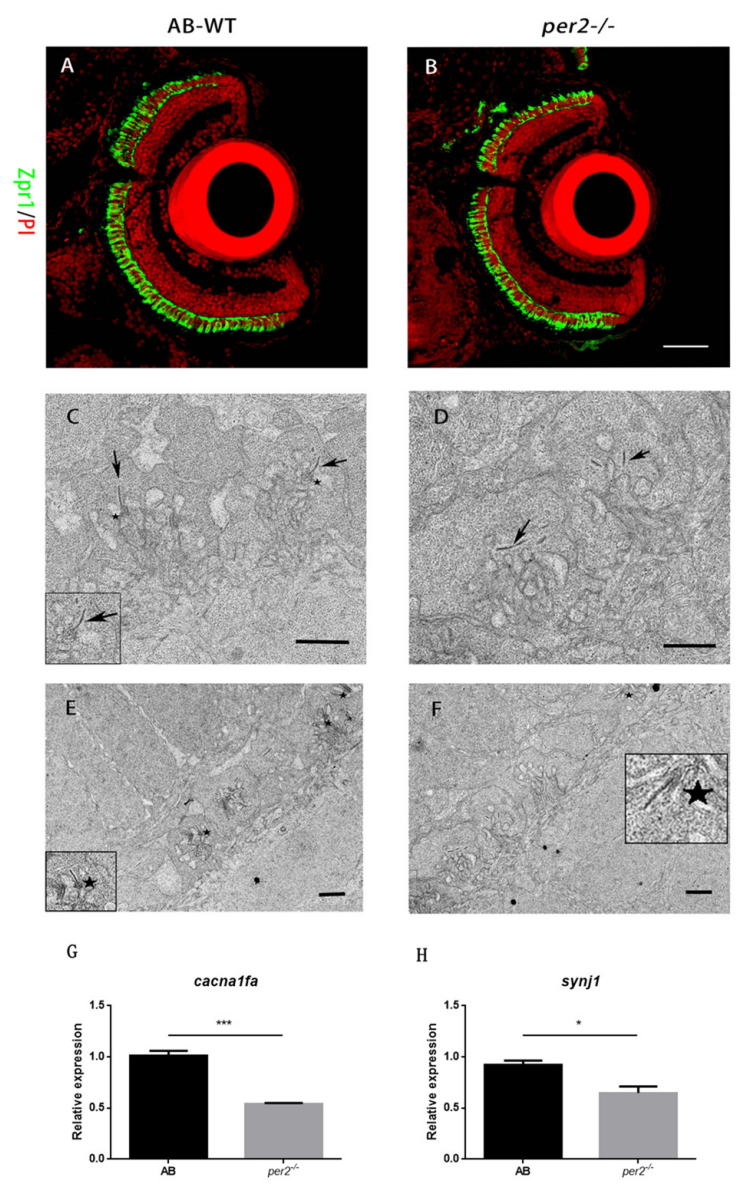

FIGURE 3 | per2 mutant zebrafish larvae exhibited normal retinal morphology and abnormal photoreceptor ribbon synapses. (A,B) AB-WT and per2 ${ }^{-1-}$ zebrafish larval retinas revealed relatively normal retinal morphology by $\mathrm{ICC}$. Images are LSCM presentations. Larvae at $5 \mathrm{dpf}$ were stained with zpr1 (green) and propidium iodide (PI; red), which label double cones and nuclei ( $n=6$ animals per genotype). Scale bar $=40 \mu \mathrm{m}$. (C-F) per2 mutant zebrafish larvae exhibited abnormal ribbon synapses. (C) In the AB-WT retina, synaptic ribbons (arrows) are associated with the presynaptic membrane via an arciform density (asterisks). (D) In the per2 ${ }^{-/-}$retina, synaptic ribbons in most of the pedicles appear to be floating in the cytoplasm and are unassociated with an arciform density and the presynaptic membrane. AB-WT (E) had relatively more normal ribbon synapses (asterisks) than those of the per2 mutant (F; $n=3$ retina per genotype). Scale bar $=1 \mu \mathrm{m}$. $(\mathbf{G}, \mathbf{H})$ Expression of cacnf1a and syjn 1 was reduced in mutant compared with WT retinas (5 dpf, $n=10$ animals per genotype, ${ }^{*} P<0.05$ and ${ }^{* * *} P<0.001$, unpaired two-tailed $t$-tests). Data represent the mean \pm SEM.

mutants. These results suggested that per2 is required for the expression of cacnalfa and synj1, which promote synaptic ribbon formation.

TABLE 2 | Ultrastructural characteristics of cone pedicles from per2 mutants and wild-type $(\mathrm{WT})$ retinas.

\begin{tabular}{lcc}
\hline & Wild-type & per2 $^{-/-}$ \\
\hline Pedicles & 40 & 40 \\
Synaptic ribbons & 56 & 62 \\
Ribbons/pedicle & $1.40 \pm 0.55$ & $1.55 \pm 0.7$ \\
Ribbons with arciform density & $73.2 \%(41 / 56)$ & $38.7 \%(24 / 62)$
\end{tabular}



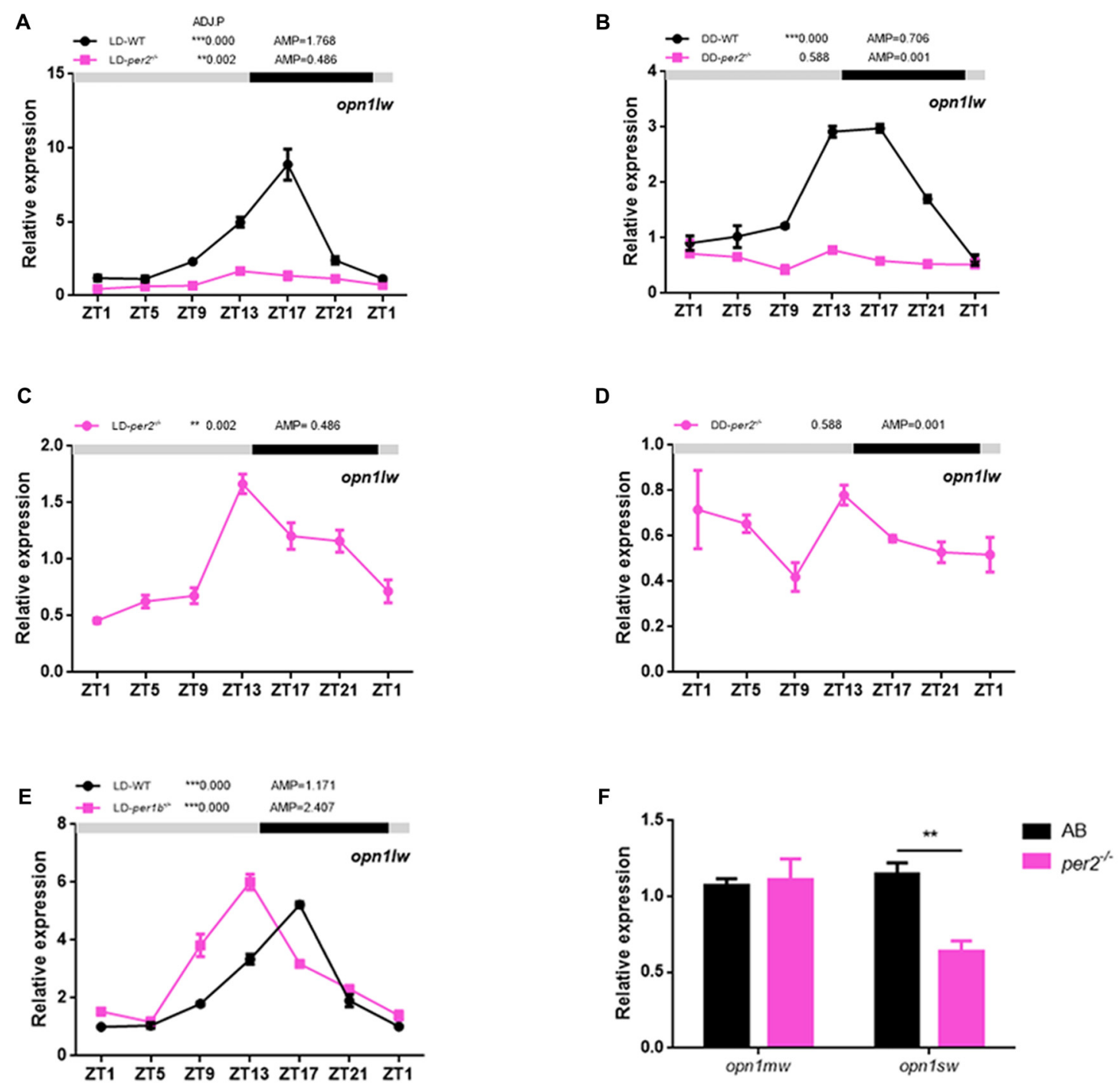

FIGURE 4 | Effects of per2 mutant on cone opsin expression. (A,B) opn1/W mRNA was attenuated in per2 ${ }^{-/-}$zebrafish larvae under the LD (Genotype: $F_{(1,2)}=393.3, P=0.0025$ ) or dark/dark (DD) condition (Genotype: $\left.F_{(1,2)}=366.9, P=0.0027\right)$. (C) Under the LD condition, the circadian rhythms of opn1/w mRNA expression remained in per2 ${ }^{-/-}$mutant zebrafish larvae (ADJ.P $=0.002$, AMP $=0.486$ ). (D) Under the DD condition, the circadian rhythms of LC opsin mRNA expression diminished in per2 $2^{-/}$zebrafish larvae (ADJ.P $=0.588$, AMP $\left.=0.001\right)$. (E) Under the LD condition, the circadian rhythms of opn $1 / \mathrm{W}$ mRNA expression between per1b mutant and WT were different (Genotype: $\left.F_{(1,2)}=153.3, P=0.0065\right)$. (F) The expression of opn $1 \mathrm{sw}$ was reduced in mutants compared with that in the WT at ZT5 (** $P<0.01$; unpaired two-tailed $t$-test). No difference in expression was detected for opn1mw. Data represent the mean \pm SEM. The mRNA expression levels were analyzed by two-way ANOVA repeated measures and the JTK-CYCLE method. ADJ.P for adjusted minimal $p$-values $(* * P<0.01$, $\left.{ }^{* * *} P<0.001\right)$, AMP for amplitude.

\section{The Expression of Cone Opsin Is Regulated by per2}

In zebrafish, the expression of long-wave-sensitive opsin (opn 1lw) mRNA fluctuates rhythmically in the day and night (Li et al., 2005, 2008). To determine whether there was a significant change in cone opsin expression in per $2^{-/-}$zebrafish, we performed quantitative real-time PCR (qRT-PCR) to detect opn1lw mRNA at different times of the day and night while the two genotypes of zebrafish embryos ( $5 \mathrm{dpf}$ ) were kept in either LD or DD conditions. opn 1 lw mRNA is only expressed in the eye (Li et al., 2008). The qRT-PCR analysis revealed a significant effect of genotype during the day in the comparison of mutants with AB-WT in either $\operatorname{LD}\left(F_{(1,2)}=393.3, P=0.0025\right.$; Figure 4A) or $\mathrm{DD}\left(F_{(1,2)}=366.9, P=0.0027\right.$; Figure $\left.4 \mathrm{~B}\right)$ conditions. In particular, per2 $2^{-/-}$zebrafish sustained the rhythm of opn1lw under the LD condition (ADJ.P $=0.002$, AMP $=0.486$, JTKCYCLE; Figure 4C) but were almost rhythm-less under the DD condition (ADJ.P $=0.588$, AMP $=0.001$; Figure 4D). However, we observed that per $1 b^{-/-}$did not dampen the rhythm of opnllw expression compared with that of the WT $\left(\right.$ per $1 b^{-/-}$: 

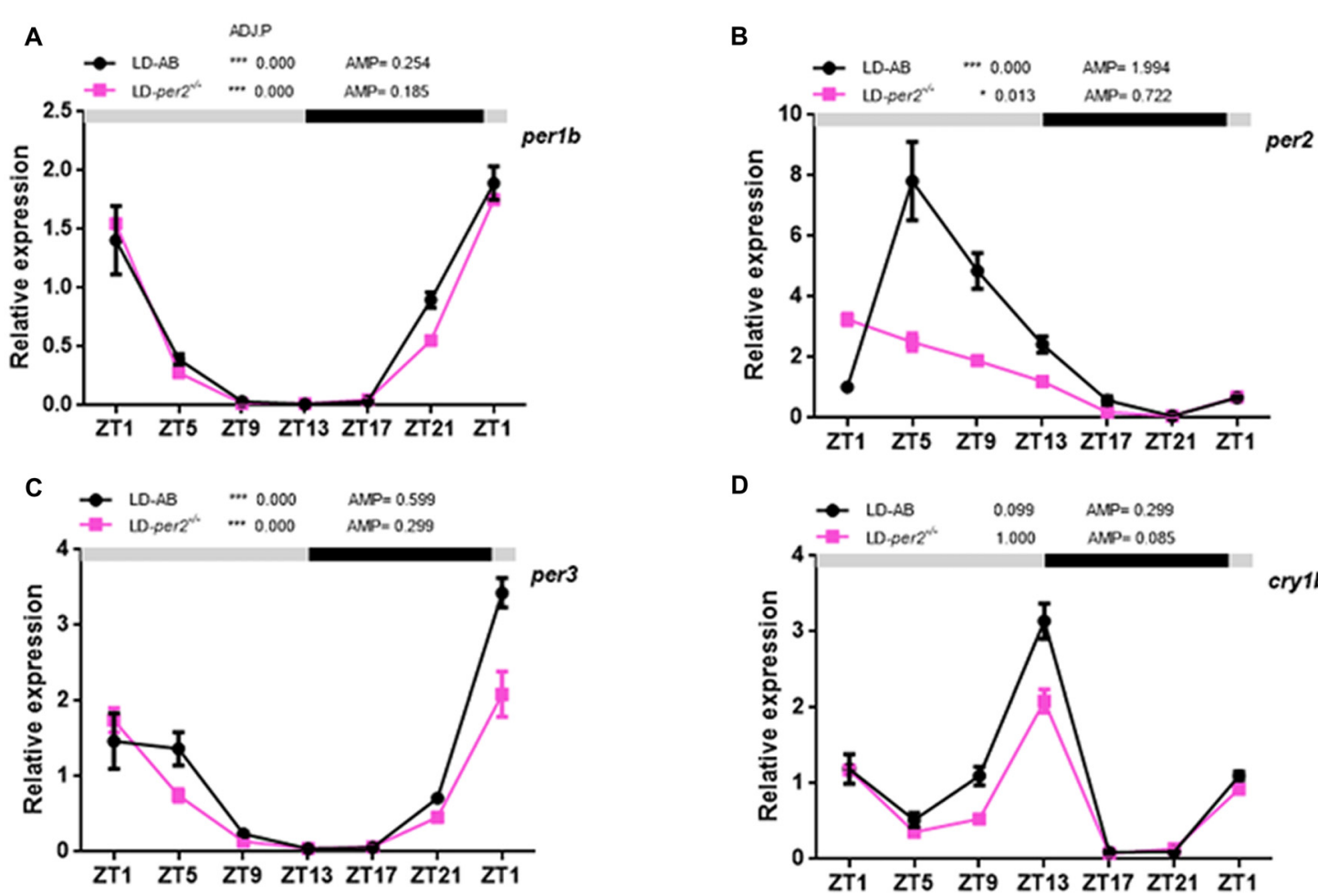

D
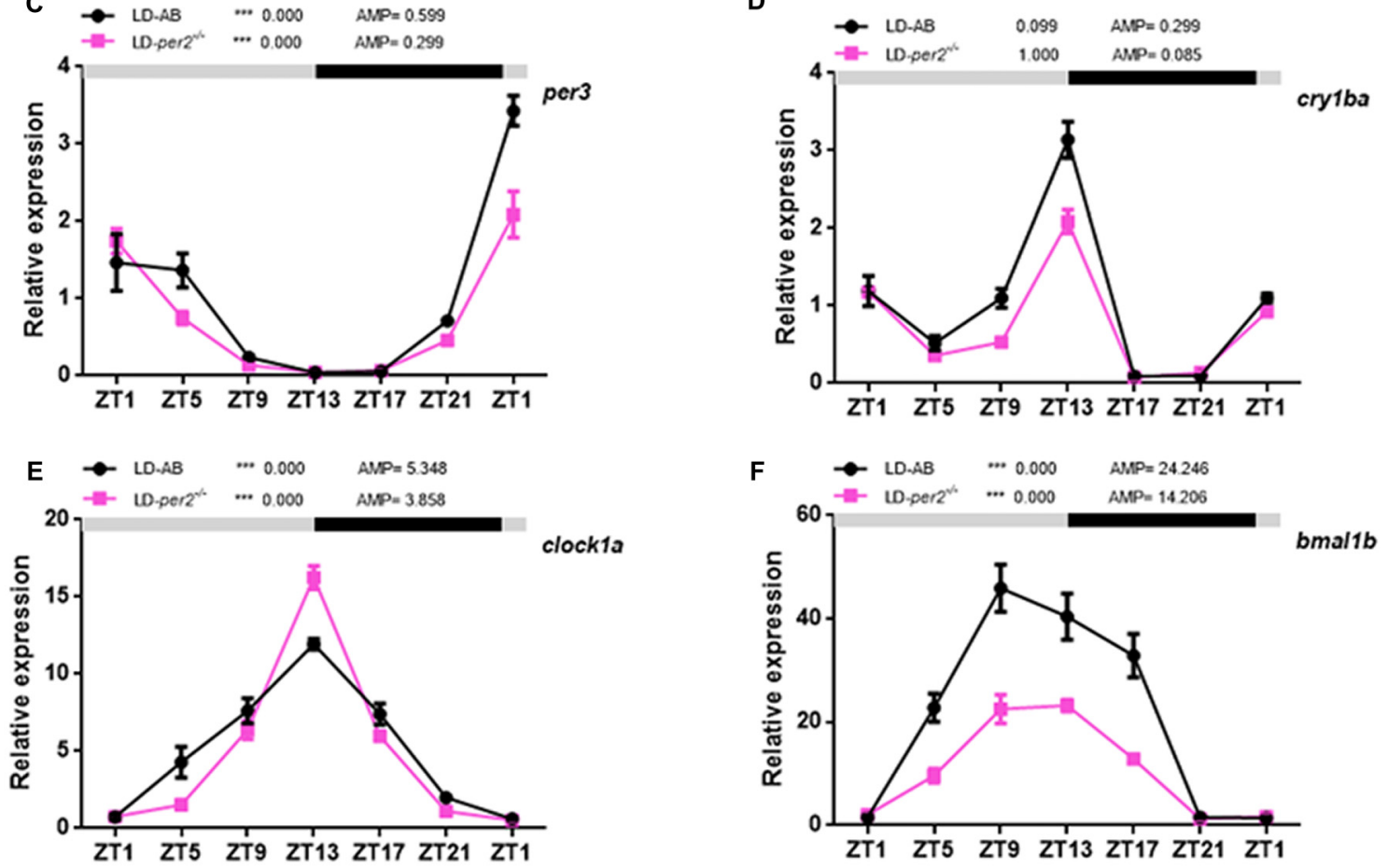

FIGURE 5 | Daily expression of mRNA of key circadian clock genes in per2 mutant adult zebrafish retinas. (A-F) WT and per2 mutant fish were sacrificed at approximately 3 months of age. Adult zebrafish retinas were dissected out and cleaned, and mRNA was quantified using real-time PCR. Each sample contained at least three female fish and three male fish. qRT-PCR showed the mRNA levels of per1b, per2, per3, clock1a, cry 1 ba and bmal1b. Two-way repeated measures ANOVA showed that per1b $\left(F_{(1,2)}=2.315, P=0.2675\right)$, per3 $\left(F_{(1,2)}=5.091, P=0.1527\right)$, and clock1a $\left(F_{(1,2)}=1.154, P=0.3951\right)$ were not affected by genotype, whereas per2 $\left(F_{(1,2)}=24.76, P=0.0381\right)$, cry 1 ba $\left(F_{(1,2)}=90.62, P=0.0109\right)$ and bmal $1 b\left(F_{(1,2)}=43.48, P=0.0222\right)$ were significantly affected by genotype. The mRNA expression levels were analyzed by two-way ANOVA repeated measures and the JTK-CYCLE method. ADJ.P for adjusted minimal $p$-values $\left({ }^{*} P<0.05\right.$, $\left.{ }^{* * *} P<0.001\right)$, AMP for amplitude. Data represent the mean $\pm \mathrm{SEM}$.

ADJ.P $<0.0001$, AMP $=1.171$ vs. WT: ADJ.P $<0.0001$, $\mathrm{AMP}=2.407$; Figure 4E), although per $1 b^{-/-}$shifted the opn 1 lw expression in the mutants. Two-way ANOVA showed significant effects of genotype $\left(F_{(1,2)}=153.3, P=0.0065\right)$. Additionally, the expression of mRNA of medium-wave-sensitive opsin (opn $1 m w)$ and short-wave-sensitive opsin (opn1sw) did not show rhythm, and that of opn1sw was significantly reduced in per2 mutants $(P=0.0026$, two-tailed unpaired $t$-test; Figure $4 \mathbf{F})$. These data indicated the important role of per 2 in cone opsin expression.

\section{The Rhythmic Expression of Clock Genes in per2 Mutant Has No Obvious Change in Adult Zebrafish Eye}

Zebrafish per2 is expressed extensively in numerous tissues (Vatine et al., 2009; Ben-Moshe et al., 2014). A previous study demonstrated that per2 expression is required for the light-induced developmental maturation of the pineal clock (Ziv et al., 2005). An assay with explanted PER2::LUC and Per1::LUC mouse retinas showed that Bmal1, Per1, Cry 1 and 
Clock are each required individually for the retina to express molecular rhythms (Ruan et al., 2012). Here, we investigated the effect of per 2 on a few core circadian oscillation genes in the adult zebrafish retina. Two-way repeated measures ANOVA showed that $\operatorname{per} 1 b\left(F_{(1,2)}=2.315, P=0.2675\right.$; Figure 5A), per3 $\left(F_{(1,2)}=5.091 ; P=0.1527 ;\right.$ Figure $\left.5 \mathrm{C}\right)$, and clock1a $\left(F_{(1,2)}=1.154, P=0.3951\right.$; Figure $\left.5 \mathrm{E}\right)$ were not affected by genotype, whereas per2 $\left(F_{(1,2)}=24.76, P=0.0381\right.$; Figure 5B), crylba $\left(F_{(1,2)}=90.62, P=0.0109\right.$; Figure 5D), and bmallb $\left(F_{(1,2)}=43.48, P=0.0222\right.$; Figure $\left.5 \mathrm{~F}\right)$ were significantly affected by genotype. In particular, the lack of expression of per2 in mutant zebrafish is primarily because erroneously synthesized per $2 \mathrm{mRNA}$ is easily degraded. Importantly, the data showed that the crylba gene obviously damped the rhythm in WT and per2 mutant zebrafish (WT: ADJ.P $=0.099$ vs. per $2^{-/-}:$ADJ.P $=1.000$; Figure 5D). In fact, this indicates the special expression pattern of the crylba gene with circadian rhythms. Therefore, per1b, per3, cry $1 b a$, clock $1 a$ and bmallb had a circadian rhythm in their gene expression. These results indicated that the per2 plays a positive role in regulation of the expression of bmallb and crylba.

\section{DISCUSSION}

Clock genes are involved in retinal processing of visual information (Storch et al., 2007; Ait-Hmyed et al., 2013; Mazzotta et al., 2013; Hakkari et al., 2016). In this study, we used per 2 mutant zebrafish larvae, which were generated using the genome-editing tool TALEN (Wang et al., 2015), and found that the gain value of OKR was reduced during the day compared with that of the $\mathrm{AB}$ WT. In a previous report, mice lacking Period 1 and Period 2 show no changes in visual responses (Ait-Hmyed et al., 2013). This difference may have occurred because per 2 has a different role in the zebrafish circadian system. Zebrafish per2 is a light-regulated gene but also has distinct regulatory functions in the different peripheral organs (Vatine et al., 2009; Wang et al., 2015). Additionally, we observed that per2 mutant zebrafish maintained an OKR circadian rhythm, which might be because mutations in per 2 do not significantly affect the rhythm of the molecular clock or the behavioral rhythm in mutant zebrafish larvae (Wang et al., 2015). We also found that mutations in per2 did not disrupt the rhythm of the molecular clock of the retina in mutants.

Moreover, per2 mutant zebrafish larvae showed a light-on VMR defect, which is a phenotype similar to that of nrc mutant zebrafish with an ON pathway defect and fewer light-driven ganglion cells (Emran et al., 2007). Our data showed that per 2 mutants exhibited abnormal and decreased arciform ribbon synapses (Figures 3D,F), and these arciform ribbon synapses are mainly formed between $\mathrm{ON}$ bipolar and horizontal cells. As mentioned previously, wud mutants display abnormal ERGs and cone synaptic ribbon formation is defective (Jia et al., 2014). This finding indicates that a synaptic ribbon defect could account for the attenuated visual function. Additionally, a previous study demonstrated that per $1 b$ mutant zebrafish eyes exhibit retinal dopaminergic deficiency
(Nie et al., 2018), and the expression of genes that play roles in specification, differentiation, and development or maintenance of dopaminergic neurons is significantly downregulated in per $1 b$ mutants (Huang et al., 2015). However, the question of whether the characteristics of per2 mutants are showed up in single or double mutations in other clock genes requires further study.

We also examined the possible effects of zebrafish per2 on the expression levels of cone opsin because the fluctuation of behavioral red cone sensitivity correlates with circadian expression of opn1lw during the first $24 \mathrm{~h}$ of constant conditions ( $\mathrm{Li}$ et al., 2005). Ait-Hmyed reported that cone opsin mRNA is reduced in mice lacking Period 1 and Period 2 (Ait-Hmyed et al., 2013). Moreover, the clock gene is required to maintain the circadian rhythms of opn1lw expression, and the circadian rhythms of opn1lw expression are diminished by anti-clock morpholinos in zebrafish embryos (Li et al., 2008). Our results showed that per2 gene mutants significantly down regulated opn $1 \mathrm{l} w$ expression, with a 3 -fold decrease, and diminished the rhythm of opn1lw expression under the DD condition. These results suggested that opn1lw mRNA is mediated by light and by an endogenous circadian mechanism. According to a previous report, CLOCK may regulate the circadian rhythms of opn1lw expression via cAMP in the zebrafish retina (Li et al., 2008). Thus, we hypothesized that PER2 as another clock gene might regulate the circadian rhythms of opn 1lw expression via cAMP signaling pathways. However, per3 expression significantly decreased in anti-Clock morpholine embryos ( $\mathrm{Li}$ et al., 2008), and the per2 mutant genotype did not significantly affect clockla gene expression in the retina (Figure 5E). Moreover, CLOCK mutation can damp the circadian expression of the clock gene mPer2 in mouse SCN and liver (Oishi et al., 2005; Vitaterna et al., 2006). Combining the above evidence, we hypothesized that per 2 might be downstream of CLOCK to control the opn 1 lw expression. Further studies will address these questions.

In a previous report, Per1 and Per2 single gene deletion mice showed different responses to photic conditions (Zheng et al., 2001), and Per $2^{\text {Brdm } 1}$ showed a progressive loss of rhythmic behavior. Ait-Hmyed did not examine single Per gene mutant mice, so we do not currently know whether blue cone deficits are in mper1 mutant or mper 2 mutant strains (Ait-Hmyed et al., 2013). In this study, we found that zebrafish per $1 b^{-/-}$and per $2^{-/-}$had different effects in regulating opn $1 l w$ mRNA expression. Short-wave-sensitive opsin (opn1sw) was also down regulated in per 2 mutants. These data suggest that different clock genes have different roles in visual function.

A recent study showed that zebrafish per2 is important for the circadian clock (Wang et al., 2015). Per2 mutant zebrafish displayed reduced activities under $\mathrm{LD}$ and a 2-h phase delay and a 1.1-h prolonged period under DD conditions (Wang et al., 2015). To determine whether per2 is essential for the expression of molecular circadian rhythms in the retina, we examined the expression of key clock genes in adult mutant retinas. We found that bmallb was down regulated 
in per2 mutants under the $\mathrm{LD}$ condition (Figure $4 \mathbf{F}$ ). In a previous report, Bmal1 regulated retinal visual processing in the retina, and a b-wave was reduced in Ret-Bmal1 ${ }^{-/-}$ mice (Storch et al., 2007). Thus, our results indicated that bmal1b might also have an important influence on vision in zebrafish.

Our previous study demonstrated that circadian misalignment does not lead to contrast defects during early development in zebrafish larvae (Nie et al., 2018). Under a constant DD conditions, per2 gene was significantly reduced in zebrafish larvae (Jensen et al., 2012), and zebrafish larvae had normal contrast sensitivity compared with that of the WT larvae (Nie et al., 2018). In this study, per2 mutant zebrafish larvae displayed reduced vision behavior due to the influences on arciform ribbon synapses, although the rhythm of clock genes was normal in the retina. These data provide evidence that clock genes affect visual function mainly by affecting neurodevelopment and not via circadian misalignment. Finally, this study implicates a major role for per2 in modulating visual information processing.

\section{REFERENCES}

Ait-Hmyed, O., Felder-Schmittbuhl, M. P., Garcia-Garrido, M., Beck, S., Seide, C., Sothilingam, V., et al. (2013). Mice lacking Period 1 and Period 2 circadian clock genes exhibit blue cone photoreceptor defects. Eur. J. Neurosci. 37, 1048-1060. doi: 10.1111/ejn.12103

Allwardt, B. A., Lall, A. B., Brockerhoff, S. E., and Dowling, J. E. (2001). Synapse formation is arrested in retinal photoreceptors of the zebrafish nrc mutant. J. Neurosci. 21, 2330-2342.

Ben-Moshe, Z., Alon, S., Mracek, P., Faigenbloom, L., Tovin, A., Vatine, G. D., et al. (2014). The light-induced transcriptome of the zebrafish pineal gland reveals complex regulation of the circadian clockwork by light. Nucleic Acids Res. 42, 3750-3767. doi: 10.1093/nar/gkt1359

Besharse, J. C. (1982). The daily light-dark cycle and rhythmic metabolism in the photoreceptor-pigment epithelial complex. Prog. Retin. Res. 1, 81-124. doi: 10.1016/0278-4327(82)90005-0

Cahill, G. M. (2002). Clock mechanisms in zebrafish. Cell Tissue Res. 309, 27-34. doi: 10.1007/s00441-002-0570-7

Douglas, R. H., Wagner, H. J., Zaunreiter, M., Behrens, U. D., and Djamgoz, M. B. A. (1992). The effect of dopamine depletion on light-evoked and circadian retinomotor movements in the teleost retina. Vis. Neurosci. 9, 335-343. doi: 10.1017/s0952523800010749

Easter, S. S. Jr., and Nicola, G. N. (1996). The development of vision in the zebrafish (Danio rerio). Dev. Biol. 180, 646-663. doi: 10.1006/dbio.1996.0335

Emran, F., Rihel, J., Adolph, A. R., Wong, K. Y., Kraves, S., and Dowling, J. E. (2007). OFF ganglion cells cannot drive the optokinetic reflex in zebrafish. Proc. Natl. Acad. Sci. U S A 104, 19126-19131. doi: 10.1073/pnas. 0709337104

Emran, F., Rihel, J., and Dowling, J. E. (2008). A behavioral assay to measure responsiveness of zebrafish to changes in light intensities. J. Vis. Exp. 20:e923. doi: $10.3791 / 923$

Hakkari, O. A. H., Acar, N., Savier, E., Spinnhirny, P., Bennis, M., FelderSchmittbuhl, M. P., et al. (2016). Rev-Erb modulates retinal visual processing and behavioral responses to light. FASEB J. 30, 3690-3701. doi: 10.1096/fj. $201600414 \mathrm{r}$

Haug, M. F., Biehlmaier, O., Mueller, K. P., and Neuhauss, S. C. F. (2010). Visual acuity in larval zebrafish: behavior and histology. Front. Zool. 7:8. doi: 10.1186/1742-9994-7-8

Huang, J., Zhong, Z., Wang, M., Chen, X., Tan, Y., Zhang, S., et al. (2015). Circadian modulation of dopamine levels and dopaminergic neuron development contributes to attention deficiency and hyperactive behavior. J. Neurosci. 35, 2572-2587. doi: 10.1523/JNEUROSCI.2551-14.2015

\section{AUTHOR CONTRIBUTIONS}

DH conceived the study, carried out the experiments and helped design them, wrote the manuscript and conceived the figures. MW, WY and YM participated in the design of the studies, data collection. HW, TX, DR and BH helped design the experiments and helped in drafting the manuscript.

\section{FUNDING}

This research was supported by grants from National Natural Science Foundation of China (81790643, 31701027), Anhui Provincial Natural Science Foundation (1708085QC58), the China Postdoctoral Science Foundation (2016M602024).

\section{ACKNOWLEDGMENTS}

We thank our Core Facility Center for Life Sciences, University of Science and Technology of China for quantitative PCR instrument and transmission electron microscope.

Hughes, M. E., Hogenesch, J. B., and Kornacker, K. (2010). JTK_CYCLE: an efficient nonparametric algorithm for detecting rhythmic components in genome-scale data sets. J. Biol. Rhythms 25, 372-380. doi: 10.1177/0748730410379711

Jensen, L. D., Cao, Z., Nakamura, M., Yang, Y., Bräutigam, L., Andersson, P., et al. (2012). Opposing effects of circadian clock genes bmall and period2 in regulation of VEGF-dependent angiogenesis in developing zebrafish. Cell Rep. 2, 231-241. doi: 10.1016/j.celrep.2012.07.005

Jia, S. J., Muto, A., Orisme, W., Henson, H. E., Parupalli, C., Ju, B. S., et al. (2014). Zebrafish Cacnalfa is required for cone photoreceptor function and synaptic ribbon formation. Hum. Mol. Genet. 23, 2981-2994. doi: 10.1093/hmg/ ddu009

Ko, C. H., and Takahashi, J. S. (2006). Molecular components of the mammalian circadian clock. Hum. Mol. Genet. 15, R271-R277. doi: 10.1093/hmg/ddl207

Larison, K. D., and Bremiller, R. (1990). Early onset of phenotype and cell patterning in the embryonic zebrafish retina. Development 109, 567-576.

Li, P., Chaurasia, S. S., Gao, Y., Carr, A. L., Iuvone, P. M., and Li, L. (2008). CLOCK is required for maintaining the circadian rhythms of opsin mRNA expression in photoreceptor cells. J. Biol. Chem. 283, 31673-31678. doi: 10.1074/jbc. M803875200

Li, P., Temple, S., Gao, Y., Haimberger, T. J., Hawryshyn, C. W., and Li, L. (2005). Circadian rhythms of behavioral cone sensitivity and long wavelength opsin mRNA expression: a correlation study in zebrafish. J. Exp. Biol. 208, 497-504. doi: $10.1242 /$ jeb. 01424

Livak, K. J., and Schmittgen, T. D. (2001). Analysis of relative gene expression data using real-time quantitative PCR and the $2^{-\Delta \Delta C_{T}}$ method. Methods 25, 402-408. doi: 10.1006/meth.2001.1262

Mazzotta, G., Rossi, A., Leonardi, E., Mason, M., Bertolucci, C., Caccin, L., et al. (2013). Fly cryptochrome and the visual system. Proc. Natl. Acad. Sci. U S A 110, 6163-6168. doi: 10.1073/pnas.1212317110

Mueller, K. P., and Neuhauss, S. C. F. (2010). Quantitative measurements of the optokinetic response in adult fish. J. Neurosci. Methods 186, 29-34. doi: 10.1016/j.jneumeth.2009.10.020

Nie, K., Wang, K., Huang, D. F., Huang, Y. B., Yin, W., Ren, D. L., et al. (2018). Effects of circadian clock protein Perlb on zebrafish visual functions. Chronobiol. Int. 35, 160-168. doi: 10.1080/07420528.2017.1391276

Oishi, K., Shirai, H., and Ishida, N. (2005). CLOCK is involved in the circadian transactivation of peroxisome-proliferator-activated receptor $\alpha(\operatorname{PPAR} \alpha)$ in mice. Biochem. J. 386, 575-581. doi: 10.1042/BJ20041150

Ren, D. L., Li, Y. J., Hu, B. B., Wang, H., and Hu, B. (2015). Melatonin regulates the rhythmic migration of neutrophils in live zebrafish. J. Pineal Res. 58, 452-460. doi: $10.1111 /$ jpi. 12230 
Reppert, S. M., and Weaver, D. R. (2002). Coordination of circadian timing in mammals. Nature 418, 935-941. doi: 10.1038/nature00965

Rinner, O., Rick, J. M., and Neuhauss, S. C. F. (2005). Contrast sensitivity, spatial and temporal tuning of the larval zebrafish optokinetic response. Invest. Ophthalmol. Vis. Sci. 46, 137-142. doi: 10.1167/iovs.04-0682

Rozen, S., and Skaletsky, H. (2000). Primer3 on the WWW for general users and for biologist programmers. Methods Mol. Biol. 132, 365-386. doi: 10.1385/159259-192-2:365

Ruan, G. X., Gamble, K. L., Risner, M. L., Young, L. A., and McMahon, D. G. (2012). Divergent roles of clock genes in retinal and suprachiasmatic nucleus circadian oscillators. PLoS One 7:e38985. doi: 10.1371/journal.pone.0038985

Storch, K.-F., Paz, C., Signorovitch, J., Raviola, E., Pawlyk, B., Li, T., et al. (2007). Intrinsic circadian clock of the mammalian retina- importance for retinal processing of visual information. Cell 130, p730-p741. doi: 10.1016/j.cell.2007. 06.045

Tamai, T. K., Young, L. C., and Whitmore, D. (2007). Light signaling to the zebrafish circadian clock by Cryptochrome 1a. Proc. Natl. Acad. Sci. U S A 104, 14712-14717. doi: 10.1073/pnas.0704588104

Tosini, G., Pozdeyev, N., Sakamoto, K., and Iuvone, P. M. (2008). The circadian clock system in the mammalian retina. Bioessays 30, 624-633. doi: 10.1002/bies. 20777

Vatine, G., Vallone, D., Appelbaum, L., Mracek, P., Ben-Moshe, Z., Lahiri, K., et al. (2009). Light directs zebrafish period2 expression via conserved D and E boxes. PLoS Biol. 7:e1000223. doi: 10.1371/journal.pbio.1000223

Vitaterna, M. H., Ko, C. H., Chang, A. M., Buhr, E. D., Fruechte, E. M., Schook, A., et al. (2006). The mouse Clock mutation reduces circadian pacemaker amplitude and enhances efficacy of resetting stimuli and phase-response curve amplitude. Proc. Natl. Acad. Sci. U S A 103, 9327-9332. doi: 10.1073/pnas. 0603601103
Wagner, H. J. (1973). Darkness-induced reduction of number of synaptic ribbons in fish retina. Nat. New Biol. 246, 53-55. doi: 10.1038/newbio246053a0

Wang, M., Zhong, Z., Zhong, Y., Zhang, W., and Wang, H. (2015). The zebrafish period 2 protein positively regulates the circadian clock through mediation of retinoic acid receptor (RAR)-related orphan receptor $\alpha$ (Ror $\alpha)$. J. Biol. Chem. 290, 4367-4382. doi: 10.1074/jbc.M114.605022

Zhang, L. Y., Xiang, L., Liu, Y. W., Venkatraman, P., Chong, L., Cho, J., et al. (2016). A naturally-derived compound schisandrin B enhanced light sensation in the pde6c zebrafish model of retinal degeneration. PLoS One 11:e0149663. doi: 10.1371/journal.pone.0149663

Zheng, B. H., Albrecht, U., Kaasik, K., Sage, M., Lu, W. Q., Vaishnav, S., et al. (2001). Nonredundant roles of the mPer1 and mPer2 genes in the mammalian circadian clock. Cell 105, 683-694. doi: 10.1016/s0092-8674(01)00380-4

Ziv, L., Levkovitz, S., Toyama, R., Falcon, J., and Gothilf, Y. (2005). Functional development of the zebrafish pineal gland: light-induced expression of period 2 is required for onset of the circadian clock. J. Neuroendocrinol. 17, 314-320. doi: 10.1111/j.1365-2826.2005.01315.x

Conflict of Interest Statement: The authors declare that the research was conducted in the absence of any commercial or financial relationships that could be construed as a potential conflict of interest.

Copyright (c) 2018 Huang, Wang, Yin, Ma, Wang, Xue, Ren and Hu. This is an open-access article distributed under the terms of the Creative Commons Attribution License (CC BY). The use, distribution or reproduction in other forums is permitted, provided the original author(s) and the copyright owner are credited and that the original publication in this journal is cited, in accordance with accepted academic practice. No use, distribution or reproduction is permitted which does not comply with these terms. 